\title{
In silico multi-epitope vaccine against covid19 showing effective interaction with HLA-B*15:03
}

Muniba Faiza1*, Tariq Abdullah², Jose Franklin Calderon-Tantalean ${ }^{3}$, Manish Ravindranath Upadhyay ${ }^{4}$, Abdelrahman H. Abdelmoneim ${ }^{5 \#}$, Fareeha Akram ${ }^{6}$, Bhupender Singh Thakur7, Ibrahim Abdulaziz ${ }^{8}$, Chimaobi James Ononamadu', Dina Abdelazim Ghoraba $^{10}$, Saba Munawar11, MD Fakhrul Islam Faruque ${ }^{12}$, Collins Kigen ${ }^{13}$, Abhishek Sharma ${ }^{14}$, Ashwani Kumar15, Aqsa Khalid ${ }^{12}$, Ali Gharip ${ }^{12}$, Ankit Gupta12, Manne Manikumar ${ }^{16}$, Uma Chaudhary ${ }^{17}$

1. Bioinformatics Review, IQL Technologies Pvt. Ltd., New Delhi, 110025, India; muniba@bioinformaticsreview.com

2. Editor, Bioinformatics Review, IQL Technologies Pvt. Ltd., New Delhi, 110025, India; tariq@bioinformaticsreview.com

3. Department of parasitology, Institute of biomedical sciences, University of São Paulo, São Paulo, Brazil; jcalderonta@usp.br

4. M.Sc. Bioinformatics, Department of Bioinformatics, G.N.I.R.D. Guru Nanak Khalsa College Nathalal Parekh Marg, Matunga East, Mumbai, Maharashtra, India; upadhyaymanish151@gmail.com

5. M.D. Clinical Immunology resident, Sudan Medical Specialization Board, Sudan; abduhamza099@gmail.com

6. BS Bioinformatics, Department of Computer Science, University of Agriculture, Faisalabad, Pakistan; farimughal869@gmail.com

7. M.Sc. School of Bioengineering and Biosciences, Lovely professional University, Punjab, India; bhupender.thakur855@gmail.com

8. B. Tech. Biochemistry, School of Life Science, Modibbo Adama University of Technology, Yola, Nigeria; inyass99@gmail.com

9. Lecturer, Department of Biochemistry and Forensic Science, Nigeria Police Academy Wudil, PMB 3474, Kano State, Nigeria; ononamaducj0016@gmail.com

10. Department of Neurosurgery, Kasr Alainy Medical School Teaching Hospitals, Faculty of Medicine and University Hospitals, Cairo University, Cairo, Egypt; dina-gh@hotmail.com

11. Research Centre for Modeling and Simulations, National University of Science and Technology Pakistan; sba.munawar@rcms.nust.edu.pk

12. Bioinformatics Review- nCoV-2019 Drug Development Team

13. Department of Biochemistry, Jomo Kenyatta University of Agriculture and Technology, Juja, Kenya; ckigen.ck@gmail.com

14. Research Scholar, Centre for Systems Biology and Bioinformatics Panjab University Chandigarh, INDIA; abhisheksharma097@gmail.com

15. Research Associate, CSIR-IHBT, Palampur, H. P-176061; ashwanibitian@gmail.com

16. NIN-TATA Centre for excellence in public health nutrition, ICMR-National Institute of Nutrition, Jamai-Osmania (Post), Hyderabad, Telangana, INDIA; mannemunikumar.bioinfo@gmail.com

17. Bhaskaracharya College of Applied Sciences, University of Delhi, Delhi, INDIA; uma.chaudhry@bcas.du.ac.in

\section{*Corresponding Author:}

Dr. Muniba Faiza

Editor-in-Chief, Bioinformatics Review, IQL Technologies Pvt. Ltd.

Email: muniba@bioinformaticsreview.com 


\section{$47 \quad$ Abstract}

The recent outbreak of severe acute respiratory syndrome (SARS) coronavirus (CoV)-2 (SARS-CoV-2) causing coronavirus disease (covid19) has posed a great threat to human health. Previous outbreaks of SARS-CoV and Middle East respiratory Syndrome CoV (MERS$\mathrm{CoV}$ ) from the same CoV family had posed similar threat to human health and economic growth. To date, not even a single drug specific to any of these CoVs has been developed nor any anti-viral vaccine is available for the treatment of diseases caused by CoVs. Subunits present in spike glycoproteins of SARS-CoV and SARS-CoV-2 are involved in binding to human ACE2 Receptor which is the primary method of viral invasion. As it has been observed in the previous studies that there are very minor differences in the spike glycoproteins of SARS-CoV and SARS-CoV-2. SARS-CoV-2 has an additional furin cleavage site that makes it different from SARS-CoV (Walls et al., 2020). In this study, we have analyzed spike glycoproteins of SARS-CoV-2 and SARS-CoV phylogenetically and subjected them to selection pressure analysis. Selection pressure analysis has revealed some important sites in SARSCoV-2 and SARS-CoV spike glycoproteins that might be involved in their pathogenicity. Further, we have developed a potential multi-epitope vaccine candidate against SARS-CoV2 by analyzing its interactions with HLA-B*15:03 subtype. This vaccine consists of multiple T-helper (TH) cells, B-cells, and Cytotoxic T-cells (CTL) epitopes joined by linkers and an adjuvant to increase its immunogenicity. Conservation of selected epitopes in SARS, MERS, and human hosts, suggests that the designed vaccine could provide cross-protection. The vaccine is designed in silico by following a reverse vaccinology method acknowledging its antigenicity, immunogenicity, toxicity, and allergenicity. The vaccine candidate that we have 
69 designed as a result of this work shows promising result indicating its potential capability of

70 simulating an immune response.

71 Keywords

SARS-CoV, SARS-COV-2, covid19, spike glycoproteins, phylogenetics, selection pressure, multi-epitope, vaccine, immune simulation.

\section{Introduction}

Coronavirus $(\mathrm{CoV})$ is a pathogen that affects the human respiratory system. Previous severe outbreaks of CoVs have been emerged posing a great threat to public health. These

77 outbreaks include Severe acute respiratory syndrome CoV (SARS-CoV) and Middle East

78 Respiratory Syndrome CoV (MERS-CoV). Recently, in December 2019, a new type of

79 coronavirus has emerged called SARS-CoV-2 or novel CoV-2019 (nCoV-2019) from Hubei

80 province in China (Zhu et al., 2020). Since then, SARS-CoV-2 has posed a great threat to public

81 health through coronavirus disease (covid19) (Pedersen \& Ho, 2020; Velavan \& Meyer,

82 2020). Due to the lack of proper antiviral treatment and vaccination, covid19 is spreading

83 person to person at a very fast rate without being an air-borne disease (Jin et al., 2020;

84 Rothan \& Byrareddy, 2020).

There is no proper treatment available for covid19 except a few FDA approved drugs including Remdesivir (Agostini et al., 2018), Favipirar ("Favipiravir shows good clinical

87 efficacy in treating COVID-19: official - Xinhua | English.news.cn," 2020), Hydrochloroquine 
treatment of covid19 yet. Continuing outbreaks of CoVs pose a great threat to human health

91 and still, there are no licensed vaccines for gaining protection against these CoVs are

92 available yet. However, there have been some attempts to develop a few vaccines for SARS-

93 CoV-2 including monoclonal antibody therapy (Shanmugaraj, Siriwattananon, Wangkanont,

94 \& Phoolcharoen, 2020) and a possible mRNA-SARS-CoV-2 vaccine (F. Wang, Kream, \&

95 Stefano, 2020). That calls for a desperate need for an anti-viral treatment and vaccine for

96 SARS-CoV-2.

In this work, we have studied spike glycoproteins of SARS-CoV-2 as they have a furin cleavage site which is conserved amongst the 144 SARS-CoV-2 isolates (Zhou et al., 2020). This cleavage site located at the boundary of S1/S2 subunits differentiates SARS-CoV-2 from SARS-CoV and other related CoVs (Walls et al., 2020). Therefore, we have analyzed spike glycoproteins phylogenetically, subjected them to selection pressure analysis, and predicted epitopes for vaccine development. We have designed an in silico novel multi-epitope potential vaccine for SARS-CoV-2. The potential epitopes were predicted from spike glycoprotein sequences. These epitopes showing immunogenicity and antigenicity were selected. They were further filtered using molecular docking with HLA-B*15:03 allele as it has been reported to be responsive in inducing an immunogenic response effectively against SARS-CoV-2 (A. Nguyen et al., 2020). The interaction of constructed multi-epitope vaccine with HLA-B*15:03 allele was analyzed using molecular docking and showed various highaffinity bonds. Furthermore, the immune response of the candidate vaccine was simulated in silico and showed a significant immune response. 


\section{Results}

112 Phylogenetic analysis

113 The constructed ML tree is shown in Figure 1. The phylogenetic tree of spike 114 glycoproteins of SARS-CoV and SARS-CoV-2 showed distinct clades of these protein groups. 115 However, the receptor-binding domain (RBD) in spike glycoprotein sequences (gi_1824676380_pdb_6W41_C and gi_1822249606_pdb_6M0J_E) of SARS-CoV-2 show

117 larger branch lengths than the other sequences of the same. The low scoring sequences were 118 eliminated from the rest of the sequences based on the phylogenetic tree. This resulted in a 119 set of relevant spike glycoprotein sequences which were further subjected to selection analysis followed by antigenic sequence identification and in silico vaccine design.

Selection pressure analysis

Spike glycoproteins of SARS-CoV-2 show positive selection

The selection pressure analysis of spike glycoprotein sequences of SARS-CoV-2 showed

124 positive selection on a few sites. However, no single site has been identified which has

127 p-value threshold of 0.05 and four such sites were identified by FUBAR (two sites coinciding 128 with MEME) with a posterior probability of 0.9 (Additional file 1). Out of these four unique 129 identified sites, two sites were found to be potentially relevant. These two sites, Cys538 and 130 Thr549, were mapped on the structure of SARS-CoV-2 spike glycoprotein (PDB ID: 6VXX) 131 (Figure 2). As evident from the results shown in Figure 2, these sites were found to be 132 present on $\beta$-sheets. The intermolecular interactions among $\beta$-sheets in a folded structure 
133 are considered important and have long been recognized (Nowick, 2008). They are involved

134 in protein-protein interaction, protein quaternary structure, and protein aggregation. $\beta$ -

135 sheet interactions in some biological processes have been considered as potential targets for

136 the treatment of diseases including cancer (J. Wang, Li, \& Jasti, 2018) and AIDS (Li et al.,

137 2013). Therefore, the two sites identified as positively selected sites may be relevant in spike

138 glycoprotein structure stability and formation.

The selection pressure analysis of spike glycoprotein sequences of SARS-CoV showed 141 positive selection on a larger number of sites as compared to that of SARS-CoV-2. However,

142 like SARS-CoV-2 spike glycoproteins, no single site has been identified which has 143 experienced negative selection. Unlike, SARS-CoV-2, BUSTED found evidence of gene-wide

144 episodic diversifying selection at a p-value <=0.05 with synonymous rate variation. FUBAR 145 has identified 2 sites of episodic positive selection with a posterior probability of 0.9 and 146 MEME has identified 9 such sites at a p-value threshold of 0.05 (Additional file 2). These 147 identified sites under positive selection were mapped on the crystal structure of spike 148 glycoprotein of SARS-CoV (PDB ID: 5X58) to recognize their relevance. The well-studied 149 structure of SARS-CoV spike glycoprotein (Walls et al., 2020) helped to locate the positively 150 selected sites in different regions of the structure. The mapping revealed that a site Thr244 151 lies in N-terminal domain (NTD), Val594 and Ala609 lie in Subdomain2 (SD2), Thr743 and 152 Pro794 lie in Linker (L) and upstream helix (UH) region, Leu803 lies in Fusion peptide (FP) 153 region, and Leu1031 lies in the central helix (CH), $\beta$-hairpin (BH), and Subdomain3 (SD3) 154 region of SARS-CoV spike glycoprotein (Figure 3). 


\section{In silico design of multi-epitope vaccine}

A complete scheme of in silico vaccine design is shown in Figure 4 and explained in the following sections.

\section{Antigenic sequences from SARS-CoV-2 spike glycoproteins}

15 antigenic high scoring (threshold $>0.5$ ) sequences were obtained out of 23 sequences of SARS-CoV-2 spike glycoprotein submitted to the VaxiJen server. These selected sequences were used to identify T-cell, B-cell, and CTL epitopes.

\section{Epitopes prediction from antigenic sequences of SARS-CoV-2 spike glycoproteins}

The total number of T-cell, B-cell, and CTL predicted epitopes were 94, 89, and 47 respectively. The predicted epitopes were further analyzed for their immunogenicity. This provided 24, 15, and 7 T-cell, B-cell, and CTL epitopes with potential immunogenicity respectively. These epitope sequences were further subjected to toxicity analysis. All these resultant sequences showed non-toxic behavior and were subjected to further analyses.

\section{Structure prediction of HLA-B*15:03 subtype}

Swissmodel provides a quality estimation of predicted models using GMQE (Studer et al., 2020) and QMEAN score (Benkert, Biasini, \& Schwede, 2011). GMQE (Global Model Quality Estimation) ranges between 0 and 1, a higher number implies higher reliability. QMEAN Z-score provides an estimate of experimental structures having a similar size. Positive values of QMEAN around zero indicate models with high quality. The HLA-B*15:03 subtype of HLA-B*15 allele with GMQE score of 0.78 and QMEAN of 0.81 (Figure 5A). Both the values indicate the predicted structure of the HLA-B*15:03 subtype is of high quality. The predicted structure was validated by plotting the Ramachandran plot. The plot showed 
$17794.2 \%$ residues in most favored regions, 5\% in additionally allowed regions, $0.8 \%$ in 178 generously allowed regions, and 0\% in disallowed regions (Figure 5B). This structure was 179 used for molecular docking of the selected epitopes possessing potential immunogenicity.

The selected epitope sequences were docked with HLA-B*15:03 subtype. The top five epitopes showing the lowest binding affinity were selected for further analyses. The binding affinities of the selected potential epitopes are shown in Table 1 along with their homology searched for MERS, SARS, and human host. According to the obtained docking results, these epitopes bound to the receptor effectively. This method was performed for each type of epitope (T-cell, B-cell, and CTL epitopes).

The designed multi-epitope vaccine consisted of 699 amino acid residues including linkers, adjuvant, and a 6x-His tag added at the C-terminus for purification purposes (Figure 6). This potential vaccine is non-allergen. Molecular weight is 75143.19 , theoretical $\mathrm{pI}$ is instability index is 35.14 classifying potential vaccine as stable. Predicted secondary structure of the multi-epitope vaccine candidate consists of $34 \%$ alpha-helices, $28 \%$ beta194 sheets, and 36\% coils (Figure 7). The predicted solubility of the multi-epitope vaccine was a 195 little below average value (0.45) (Figure 8). It implies that the multi-epitope is less soluble 196 in E. coli protein.

I-Tasser predicted five models, out of which model 1 was selected having a C-score of - 
199 The plot showed 69\% residues in most favored regions, $25.2 \%$ residues in additionally

200 allowed regions, 3.6\% residues in generously allowed regions, and $2.1 \%$ residues in

201 disallowed regions (Figure 9B).

The top-ranking pose was selected from HADDOCK showing Z-score of -1.9 , the most epitope vaccine candidate and HLA-B*15:03 subtype was analyzed using the PRODIGY

212 residues is provided in Table 2. Amongst them, the closely interacting residues include Ile90, 213 Lys92, Glu100, Ser155, Ala174, Gln179, Arg181, and Arg194 (Figure 10B). The molecular

214 docking of candidate multi-epitope vaccine with HLA-B*15:03 suggests that this designed 215 vaccine may be a good candidate against covid19. 
221 meantime, TH memory cells level increased until it reached 4000 on day 21 then jumped to

222 reach 9000 cells and fell gradually (Figure 11A). Most of these cells were in an active state

223 reaching a level of 3000 on day21 then increased again to 5000 cells on day 30 after

224 vaccination while few of them became tolerant anergic in the first week (Figure 11B). Active

225 regulatory TH cells started to rise from 20 at the beginning of vaccination to a level of 160

226 after 2 days then fell gradually (Figure 11C). TH1 (shown in plot as Th1) level increased to

227 reach a level of 50000 at day 21 then kept rising to reach 90000 cells by the day 27 (Figure

228 11D) showing 100\% till the end of the third month (Figure 11E) while the T regulatory (TR)

229 cells did not exceed the number of 160 cells.

\section{CTL (CD8):}

Non-memory CTL (CD8) can be seen increasing from below 1100 after day 0 to reach a

maximum level of 1130 number of cells on day 23, then falling again only to rise at day 27 (6

234 rise until they reached to a number of 1130 cells. CTL active cells were predicted to increase

235 after day 0 to reach a level of 900 at day 40 before it started to fall. Tolerant anergic cells did

236 not exceed the level of 600 throughout the simulation (Figure 12B). Dendritic cells (DC) can

237 either present antigen to MHC1 or MHC2. Here, around 200 DCs are presenting antigens to

238 MHC2 in comparison to only 10 DC cells presenting to MHC1 (Figure 12C).

\section{B-cells and antibodies}

The total B-cell number increased from 0 on day 1 to near 700 on day 21 before slowly

241 falling again (Figure 13A). Moreover, antibodies production was noticed to occur almost five

242 days after the beginning of infection with an earlier rise in Immunoglobulin-M (IgM) 
243 followed by the rise of Immunoglobulin-G (IgG). Both were noticed to fall until day 20, before

244 rising again after the second injection at day 21 (Figure 13B).

\section{Cytokines, Interleukins, and Natural killer (NK) cells:}

Cytokines are necessary for successful vaccination. Here, for our designed vaccine

247 candidate, all cytokines started to increase after day 0 to reach a maximum level at day 5 and

2487 , then started to fall, only to rise again after the second vaccine injection at day 21 to reach

249 similar levels except for Interferon-y (IFN-y) whose subsequent rise is only up to 1250 in

250 comparison to 420000 in the first injection reaction. Special focus was on the response of

251 Interleukin-2 (IL-2), where a higher level (450000) of IL-2 was noticed after the second

252 injection (Figure 14A).

NK cells are an integral part of the early response to the virus. The in silico simulation of

254 vaccine candidate showed high fluctuations in the population of NK cells throughout the 90

255 days beginning from a level of 345 cells then fluctuated to reach a level around 330 by the

256 end of third month (Figure 14B).

\section{Conclusion}

In this study, we have studied spike glycoproteins of SARS-CoV and SARS-CoV-2

259 phylogenetically and designed a multi-epitope vaccine for covid19. The selection pressure

260 analysis on spike glycoproteins revealed several important sites that have undergone

261 positive selection. Interestingly, no negative selection was found in both types of CoVs. As

262 negative selection selectively removes the harmful variants, it indicates that the selection

263 process in SARS-CoVs has not been stabilized yet. Additionally, it also suggests that the 
264 negative selection in SARS-CoVs is quite weak that is allowing the widespread of deleterious

265 mutations in the genome. This can eventually lead to the back mutations which may

266 potentially act as one of the contributing factor in maintaining the genomic integrity of the

267 virus (Loewe, 2008). Besides, unlike SARS-CoV, the gene-wide episodic diversifying selection

268 was not found in SARS-CoV-2 spike glycoproteins.

A proper methodology was followed during the in silico designing of a multi-epitope vaccine candidate. The potential epitopes were screened using molecular docking and were

271 selected based on the lowest binding affinity. The vaccine was constructed using these

272 epitopes only for better binding results. This vaccine candidate shows effective binding with

273 the HLA-B*15:03 subtype that is effective in eliciting an immune response against SARS-CoV-

274 2. The in silico immune simulation of the designed vaccine showed a significant rise in TH

275 cells after the first injection and reached its maximum level after the second injection

276 accompanied by the formation of TH memory cells. Further, the total number of CTL and DCs

277 increased over three months. IgM levels reached a high value after the second injection

278 followed by a rise in IgG levels. Furthermore, IL-2 reached its maximum value at day 7 and

279 then attained even higher levels after the second injection. These results show that the 280 designed multi-epitope vaccine candidate is capable of inducing a humoral immune response

281 against covid19. However, it still requires the experimental results and clinical trials to be 282 more assured about the same. Additionally, this designed vaccine candidate could be 283 beneficial in providing cross-protection against covid19 and the other similar viral diseases. 


\section{Methods}

285

286

287

288

289

290

291

292

293 294 the phylogenetic tree.

295

296

297

298

299

300

301

302

\section{Phylogenetic analysis}

\section{Selection Analysis} at each site respectively.

Protein sequences of SARS-CoV-2 spike glycoprotein were retrieved from NCBI (www.ncbi.nih.nlm.gov) (Table 3). A phylogenetic tree of spike glycoprotein sequences of

SARS-CoV-2 was constructed using IQTREE (L. T. Nguyen, Schmidt, Von Haeseler, \& Minh, 2015). SARS-CoV spike glycoprotein sequences were used as the outgroup and were downloaded from NCBI (www.ncbi.nih.nlm.gov). The best model used was VT+G4, namely, the 'Variable Time' matrix (Müller \& Vingron, 2001) with Gamma distribution (under four rate categories) as predicted by ModelFinder (Kalyaanamoorthy, Minh, Wong, Von Haeseler, \& Jermiin, 2017). Some of the outliner (low scoring) sequences were removed according to

The evolutionary analysis of SARS-CoV-2 spike glycoprotein sequences and GISAID sequences was carried out at the Datamonkey server (https://www.datamonkey.org) of HYPHY program (Pond \& Muse, 2005). BUSTED (Branch-site Unrestricted Statistical Test for Episodic Diversification) (Murrell et al., 2015), FUBAR (Fast Unconstrained Bayesian Approximation) (Murrell et al., 2013) and MEME (Mixed Effects Maximum Likelihood) (Murrell et al., 2012) methods were used to detect episodic and pervasive selection pressure 


\section{In silico design of multi-epitope vaccine}

\section{Antigenic sequence identification}

The antigenic sequences among the selected sequences were identified using the VaxiJen server (http://www.ddg-pharmfac.net/vaxijen/VaxiJen/VaxiJen.html). The threshold was set to the default value of 0.5 . VaxiJen predicts protective antigens with accuracy ranging between $79 \%$ to $89 \%$ as tested on three different datasets (Doytchinova \& Flower, 2007).

\section{Epitope prediction and selection}

B-cell epitopes were predicted using SVMTrip (Yao, Zhang, Liang, \& Zhang, 2012). T-cell epitopes were predicted using Tepitool (Paul, Sidney, Sette, \& Peters, 2016) of IEDB (Vita et al., 2019) with IC-50 value more than $500 \mathrm{nM}$ for MHC-I and MHC-II. CTLs were predicted using CTLPred (Bhasin \& Raghava, 2004). The predicted epitopes were subjected to immunogenicity analysis using IEDB (Vita et al., 2019). The predicted potential immunogens were analyzed for toxicity using ToxinPred (Gupta et al., 2013).

\section{Structure prediction and validation of HLA-B*15:03 subtype}

Homology modeling was performed to obtain the structures of HLA-B*15:03 subtype and selected epitopes using the Swissmodel (Waterhouse et al., 2018). The predicted structures of HLA-B*15:03 was further validated by creating the Ramachandran Plot using PROCHECK (Laskowski, MacArthur, Moss, \& Thornton, 1993).

\section{Selection of potential epitopes for multi-epitope vaccine}

The selected epitopes with potential immunogenicity were docked with HLA-B*15:03 subtype to filter out epitopes. The docking was carried out using Autodock Vina (Trott \& Olson, 2009). Binding pocket and residues in HLA-B*15:03 were identified using the CASTp 
3.0 server (Tian, Chen, Lei, Zhao, \& Liang, 2018). The binding $\mathrm{x}, \mathrm{y}$, and $\mathrm{z}$ coordinates in Autodock Vina were defined as 5.103, 13.862, and 146.304 respectively.

\section{Construction of multi-epitope vaccine}

Top 5 epitopes from each B-cell, T-cell, and CTL epitopes showing the lowest binding affinities towards HLA-B*15:03 were selected from the bound epitopes. The finally selected epitopes were joined together using GPGPG and AAY linkers. An adjuvant (Q0VDF9) was added at the $\mathrm{N}$-terminal of the multi-epitope vaccine using the EAAAK linker to increase its immunogenicity. The adjuvant sequence was downloaded from the Uniprot database (https://www.uniprot.org).

Secondary and tertiary structure prediction of multi-epitope vaccine Secondary structure was predicted by the PSIPRED server (http://globin.bio.warwick.ac.uk/psipred/) and

RaptorX (http://raptorx.uchicago.edu/StructurePropertyPred/predict/). The PSIPRED first identifies homologs using PSI-BLAST and then employs feed-forward neural networks to predict the secondary structure (McGuffin, Bryson, \& Jones, 2000). RaptorX predicts secondary structure without using any template. It incorporates deep convolutional neural fields to predict secondary structure, solvent accessibility, and disordered regions ( $\mathrm{S}$. Wang, Li, Liu, \& Xu, 2016). The ab-initio tertiary structure of designed multi-epitope was predicted using I-Tasser (Yang \& Zhang, 2015) server (https://zhanglab.ccmb.med.umich.edu/ITASSER/). The I-Tasser (Iterative threading assembly refinement) predicts accurate structures by first identifying templates from PDB using multiple threading and identifies functions through the protein functions database. It has been ranked as the best server for 
347 protein structure and function prediction in CASP experiments (Shey et al., 2019). The

348 predicted tertiary structure of the multi-epitope vaccine was validated using PROCHECK

349 (Laskowski et al., 1993).

Physicochemical properties prediction of multi-epitope vaccine

351

The allergenicity of the designed vaccine candidate was predicted using Allertop v. 2.0

(http://www.ddg-pharmfac.net/AllerTOP) and AllergenFP v. 1.0 (http://ddg-

353

354

355

356

357

358

359

360

361

362

with

HLA-B*15:03

using

HADDOCK

webserver

367 protein Docking) is an integrated platform for flexible docking and performing short 
369 docking of multi-epitope and HLA-B*15:03 was performed by defining identified active

370

371

372

373

374

375

376

377

378

379

380

381

382

383

384

385

386

387

388

389

390

391

residues. The active residues of HLA-B allele subtype predicted structure identified from CP0RT server were Gly80, Pro81, Glu82, Tyr83, Trp84, Asp85, Arg86, Glu87, Thr88, Gln89, Ile90, Ser91, Lys92, Thr93, Asn94, Thr95, Gln96, Thr97, Tyr98, Ala174, Arg175, Glu176, Ala177, Glu178, Gln179, Leu180, Arg181, Ala182, Tyr183, Leu184, Glu185, Gly186, Leu187, Cys188, Val189, Glu190, Trp191, Leu192, Arg193, Arg194, Tyr195, Leu196, Glu197, Asn198, and Gly199. Active residues for multi-epitope vaccine identified using CPORT were Tyr18, Phe281, Asp292, Ile377, Gly380, Leu385, Ser389, Leu390, Gly538, Gly542, Ala545, Glu546, Leu549, Ala550, Pro553, Gly554, Pro555, Asn559, Val560, Phe561, Ala562, Pro564, Gly566, Pro567, Gly568, Pro569, Gly570, Val571, Val572, Val573, Ala576, Asn594, His597, Ala599, Ser601, Phe604, Ala635, Cys639, Pro640, Phe641, Glu643, Val644, Phe645, Ala646, Ala647, Tyr648, Phe649, Gly650, Asp651, Asp652, Thr653, Val654, Ile655, Glu656, Val657, Ala658, Ala659, Tyr660, Phe661, Ser662, Tyr663, Phe664, Ala665, Val666, His667, Phe668, Ile669, Ala673, Val680, Val681, Ala682, Ala683, Tyr684, Lys685, Arg688, Leu691, and Ala692. The passive residues were set to be detected in surrounding surface. An MD simulation of 1000 steps of energy minimization, 100 steps for heating phase, 1250 steps at $300 \mathrm{~K}$, and 500 steps of cooling phase was performed.

\section{In silico immune simulation}

The immunogenicity and immune response of the designed multi-epitope vaccine was determined using the C-ImmSim server (http://150.146.2.1/CIMMSIM/index.php?page=1). C-ImmSim is an agent-based simulator that uses systems biology and data-driven prediction methods to predict immune response to viruses, bacteria, or a vaccine (Rapin, Lund, Bernaschi, \& Castiglione, 2010). The following most common 
412

413

414

415

416

alleles in Caucasian, Asian and Afro Brazilian were chosen HLA-A*02, 24, HLA-A*02, 03, DRB1*11, 13, DRB1*13, 11, 03 along with HLA-B*15:03 since it was shown to have great efficiency in presenting conserved SARS-CoV-2 that are shared between common human CoVs (A. Nguyen et al., 2020). The rest of the parameters were set as the following default values. Random Seed (12345) was used, simulation volume was set to 10, and simulation steps were set to 270 (=90 days) with two injections the first at the 1-time step which equals the first eight hours of simulation, and the second injection at 63-time steps which is equal to three weeks. The rest of the parameters were left with default values. No specific antigen sequence was added to the inputs.

\section{Declaration}

The authors declare that there is no conflict of interest whatsoever.

Agostini, M. L., Andres, E. L., Sims, A. C., Graham, R. L., Sheahan, T. P., Lu, X., ... Denison, M. R. (2018). Coronavirus susceptibility to the antiviral remdesivir (GS-5734) is mediated by the viral polymerase and the proofreading exoribonuclease. MBio, 9(2). https://doi.org/10.1128/mBio.00221-18

Benkert, P., Biasini, M., \& Schwede, T. (2011). Toward the estimation of the absolute quality of individual protein structure models. Bioinformatics, 27(3), 343-350. https://doi.org/10.1093/bioinformatics/btq662

Bhasin, M., \& Raghava, G. P. S. (2004). Prediction of CTL epitopes using QM, SVM and ANN techniques. Vaccine, 22(23-24), 3195-3204. https://doi.org/10.1016/j.vaccine.2004.02.005

Dimitrov, I., Bangov, I., Flower, D. R., \& Doytchinova, I. (2014). AllerTOP v.2 - A server for in silico prediction of allergens. Journal of Molecular Modeling, 20(6), 1-6. https://doi.org/10.1007/s00894-014-2278-5 
417 Dimitrov, I., Naneva, L., Doytchinova, I., \& Bangov, I. (2014). AllergenFP: Allergenicity 418 prediction by descriptor fingerprints. Bioinformatics, 30(6), 846-851. 419 https://doi.org/10.1093/bioinformatics/btt619

420 Doytchinova, I. A., \& Flower, D. R. (2007). VaxiJen: A server for prediction of protective 421 antigens, tumour antigens and subunit vaccines. BMC Bioinformatics, 8(1), 4. 422 https://doi.org/10.1186/1471-2105-8-4

Favipiravir shows good clinical efficacy in treating COVID-19: official - Xinhua | 424 English.news.cn. (2020). Retrieved May 18, 2020, from 425 http://www.xinhuanet.com/english/2020-03/17/c_138888226.htm

Gasteiger, E., Hoogland, C., Gattiker, A., Duvaud, S., Wilkins, M. R., Appel, R. D., \& Bairoch, A. (2005). Protein Identification and Analysis Tools on the ExPASy Server. In J. M. Walker (Ed.), The Proteomics Protocols Handbook (pp. 571-607). Springer. https://doi.org/10.1385/159259-890-0:571

Gautret, P., Lagier, J.-C., Parola, P., Hoang, V. T., Meddeb, L., Mailhe, M., ... Raoult, D. (2020). Hydroxychloroquine and azithromycin as a treatment of COVID-19: results of an open-label non-randomized clinical trial. International Journal of Antimicrobial Agents, 105949. https://doi.org/10.1016/j.ijantimicag.2020.105949

Gupta, S., Kapoor, P., Chaudhary, K., Gautam, A., Kumar, R., \& Raghava, G. P. S. (2013). In Silico Approach for Predicting Toxicity of Peptides and Proteins. PLoS ONE, 8(9), e73957. https://doi.org/10.1371/journal.pone.0073957

Jean, S. S., Lee, P. I., \& Hsueh, P. R. (2020). Treatment options for COVID-19: The reality and challenges. Journal of Microbiology, Immunology and Infection. https://doi.org/10.1016/j.jmii.2020.03.034

Jin, Y., Yang, H., Ji, W., Wu, W., Chen, S., Zhang, W., \& Duan, G. (2020). Virology, epidemiology, pathogenesis, and control of covid-19. Viruses, 12(4), 372. https://doi.org/10.3390/v12040372

Kalyaanamoorthy, S., Minh, B. Q., Wong, T. K. F., Von Haeseler, A., \& Jermiin, L. S. (2017). ModelFinder: Fast model selection for accurate phylogenetic estimates. Nature Methods, 14(6), 587-589. https://doi.org/10.1038/nmeth.4285

Laskowski, R. A., MacArthur, M. W., Moss, D. S., \& Thornton, J. M. (1993). PROCHECK: a program to check the stereochemical quality of protein structures. Journal of Applied Crystallography, 26(2), 283-291. https://doi.org/10.1107/s0021889892009944

Li, B. Q., Niu, B., Chen, L., Wei, Z. J., Huang, T., Jiang, M., ... Cai, Y. D. (2013). Identifying Chemicals with Potential Therapy of HIV Based on Protein-Protein and Protein-Chemical Interaction Network. PLoS ONE, 8(6), 65207. https://doi.org/10.1371/journal.pone.0065207 
461

462

463

464

465

466

467

468

469

470

471

472

473

474

475

476

477

478

479

480

481

482

483

484

485

Loewe, L. (2008). Negative Selection. Nature Education 1(1):59. Retrieved from https://www.nature.com/scitable/topicpage/negative-selection-1136/

McGuffin, L. J., Bryson, K., \& Jones, D. T. (2000). The PSIPRED protein structure prediction server. Bioinformatics (Vol. 16). https://doi.org/10.1093/bioinformatics/16.4.404

Müller, T., \& Vingron, M. (2001). Modeling amino acid replacement. Journal of Computational Biology, 7(6), 761-776. https://doi.org/10.1089/10665270050514918

Murrell, B., Moola, S., Mabona, A., Weighill, T., Sheward, D., Kosakovsky Pond, S. L., \& Scheffler, K. (2013). FUBAR: A fast, unconstrained bayesian AppRoximation for inferring selection. Molecular Biology and Evolution, 30(5), 1196-1205. https://doi.org/10.1093/molbev/mst030

Murrell, B., Weaver, S., Smith, M. D., Wertheim, J. O., Murrell, S., Aylward, A., ... Kosakovsky Pond, S. L. (2015). Gene-wide identification of episodic selection. Molecular Biology and Evolution, 32(5), 1365-1371. https://doi.org/10.1093/molbev/msv035

Murrell, B., Wertheim, J. O., Moola, S., Weighill, T., Scheffler, K., \& Kosakovsky Pond, S. L. (2012). Detecting individual sites subject to episodic diversifying selection. PLoS Genetics, 8(7), e1002764. https://doi.org/10.1371/journal.pgen.1002764

Nguyen, A., David, J. K., Maden, S. K., Wood, M. A., Weeder, B. R., Nellore, A., \& Thompson, R. F. (2020). Human leukocyte antigen susceptibility map for SARS-CoV-2. Journal of Virology. https://doi.org/10.1128/jvi.00510-20

Nguyen, L. T., Schmidt, H. A., Von Haeseler, A., \& Minh, B. Q. (2015). IQ-TREE: A fast and effective stochastic algorithm for estimating maximum-likelihood phylogenies. Molecular Biology and Evolution, 32(1), 268-274. https://doi.org/10.1093/molbev/msu300

Nowick, J. S. (2008). Exploring $\beta$-sheet structure and interactions with chemical model systems. Accounts of Chemical Research, 41(10), 1319-1330. https://doi.org/10.1021/ar800064f

Paul, S., Sidney, J., Sette, A., \& Peters, B. (2016). TepiTool: A pipeline for computational prediction of T cell epitope candidates. Current Protocols in Immunology, 2016, 18.19.118.19.24. https://doi.org/10.1002/cpim.12

Pedersen, S. F., \& Ho, Y. C. (2020). SARS-CoV-2: A storm is raging. Journal of Clinical Investigation, 130(5), 2202-2205. https://doi.org/10.1172/JCI137647

Pond, S. L. K., \& Muse, S. V. (2005). HyPhy: Hypothesis Testing Using Phylogenies. In Statistical Methods in Molecular Evolution (pp. 125-181). Springer-Verlag. https://doi.org/10.1007/0-387-27733-1_6 
Rapin, N., Lund, O., Bernaschi, M., \& Castiglione, F. (2010). Computational immunology meets bioinformatics: The use of prediction tools for molecular binding in the simulation of the immune system. PLoS ONE, 5(4), e9862. https://doi.org/10.1371/journal.pone.0009862

Rothan, H. A., \& Byrareddy, S. N. (2020). The epidemiology and pathogenesis of coronavirus disease (COVID-19) outbreak. Journal of Autoimmunity, 109, 102433. https://doi.org/10.1016/j.jaut.2020.102433

Shanmugaraj, B., Siriwattananon, K., Wangkanont, K., \& Phoolcharoen, W. (2020, March 1). Perspectives on monoclonal antibody therapy as potential therapeutic intervention for Coronavirus disease-19 (COVID-19). Asian Pacific Journal of Allergy and Immunology. NLM (Medline). https://doi.org/10.12932/AP-200220-0773

Shey, R. A., Ghogomu, S. M., Esoh, K. K., Nebangwa, N. D., Shintouo, C. M., Nongley, N. F., ... Souopgui, J. (2019). In-silico design of a multi-epitope vaccine candidate against onchocerciasis and related filarial diseases. Scientific Reports, 9(1), 1-18. https://doi.org/10.1038/s41598-019-40833-x

Studer, G., Rempfer, C., Waterhouse, A. M., Gumienny, R., Haas, J., \& Schwede, T. (2020). QMEANDisCo-distance constraints applied on model quality estimation. Bioinformatics (Oxford, England), 36(6), 1765-1771. https://doi.org/10.1093/bioinformatics/btz828

Tian, W., Chen, C., Lei, X., Zhao, J., \& Liang, J. (2018). CASTp 3.0: Computed atlas of surface topography of proteins. Nucleic Acids Research, 46(W1), W363-W367. https://doi.org/10.1093/nar/gky473

Trott, O., \& Olson, A. J. (2009). AutoDock Vina: Improving the speed and accuracy of docking with a new scoring function, efficient optimization, and multithreading. Journal of Computational Chemistry, 31(2), NA-NA. https://doi.org/10.1002/jcc.21334

Van Zundert, G. C. P., Rodrigues, J. P. G. L. M., Trellet, M., Schmitz, C., Kastritis, P. L., Karaca, E., ... Bonvin, A. M. J. J. (2016). The HADDOCK2.2 Web Server: User-Friendly Integrative Modeling of Biomolecular Complexes. Journal of Molecular Biology, 428(4), 720-725. https://doi.org/10.1016/j.jmb.2015.09.014

Velavan, T. P., \& Meyer, C. G. (2020). The COVID-19 epidemic. Tropical Medicine and International Health, 25(3), 278-280. https://doi.org/10.1111/tmi.13383

Vita, R., Mahajan, S., Overton, J. A., Dhanda, S. K., Martini, S., Cantrell, J. R., ... Peters, B. (2019). The Immune Epitope Database (IEDB): 2018 update. Nucleic Acids Research, 47(D1), D339D343. https://doi.org/10.1093/nar/gky1006

Walls, A. C., Park, Y. J., Tortorici, M. A., Wall, A., McGuire, A. T., \& Veesler, D. (2020). Structure, Function, and Antigenicity of the SARS-CoV-2 Spike Glycoprotein. Cell, 181(2), 281-292.e6. https://doi.org/10.1016/j.cell.2020.02.058 
521 Wang, F., Kream, R. M., \& Stefano, G. B. (2020). An evidence based perspective on mRNA-

522 SARScov-2 vaccine development. Medical Science Monitor, 26.

523 https://doi.org/10.12659/MSM.924700

524 Wang, J., Li, X., \& Jasti, B. (2018). Design of Beta-sheet Hairpin Peptide Targeting to B7H6 525 Protein. Research Day. Retrieved 526 https://scholarlycommons.pacific.edu/researchday/2018/events/15

527 Wang, S., Li, W., Liu, S., \& Xu, J. (2016). RaptorX-Property: a web server for protein structure 528 property prediction. Nucleic Acids Research, 44(W1), W430-W435. 529 https://doi.org/10.1093/nar/gkw306

530 Waterhouse, A., Bertoni, M., Bienert, S., Studer, G., Tauriello, G., Gumienny, R., ... Schwede, T. 531 (2018). SWISS-MODEL: Homology modelling of protein structures and complexes. Nucleic 532 Acids Research, 46(W1), W296-W303. https://doi.org/10.1093/nar/gky427

533 Yang, J., \& Zhang, Y. (2015). I-TASSER server: New development for protein structure and 534 function predictions. Nucleic Acids Research, 43(W1), W174-W181. 535 https://doi.org/10.1093/nar/gkv342

536 Yao, B., Zhang, L., Liang, S., \& Zhang, C. (2012). SVMTriP: A Method to Predict Antigenic 537 Epitopes Using Support Vector Machine to Integrate Tri-Peptide Similarity and Propensity. 538 PLoS ONE, 7(9), e45152. https://doi.org/10.1371/journal.pone.0045152

539 Zhou, P., Yang, X. Lou, Wang, X. G., Hu, B., Zhang, L., Zhang, W., ... Shi, Z. L. (2020). A pneumonia 540 outbreak associated with a new coronavirus of probable bat origin. Nature, 579(7798), 270541 273. https://doi.org/10.1038/s41586-020-2012-7

542 Zhu, N., Zhang, D., Wang, W., Li, X., Yang, B., Song, J., ... Tan, W. (2020). A novel coronavirus 543 from patients with pneumonia in China, 2019. New England Journal of Medicine, 382(8), 727544 733. https://doi.org/10.1056/NEJMoa2001017 
546

547

548

549

550

551

552

553

554

555

556

557

558

559

560

561

562

563

564

565

566

\section{Legends}

Figure 1 ML tree of spike glycoprotein sequences of SARS-CoV and SARS-CoV-2. Red sequences represent SARS-CoV-2 and green colored sequences show SARS-CoV spike glycoproteins.

Figure 2 Two sites identified as positively selected mapped on crystal structure SARSCoV-2 spike glycoprotein (PDB ID: 6VXX). The two identified sites are shown in red sticks.

Figure 3 Identified positively selected sites (red sticks) mapped on crystal structure SARS-CoV spike glycoprotein (PDB ID: 5X58). Different colors on the structure depict different regions. NTD: N-terminal domain, RBD: Receptor binding domain, SD1: Subdomain1, SD2: Subdomain2, SD3: Subdomain3, CH: Central helix, BH: $\beta$-hairpin, L: Linker, UH: Upstream helix, FP: Fusion protein, HR1: Heptad repeat1.

Figure 4 Schema of the methodology followed in the in silico design of a multi-epitope vaccine.

Figure 5 A) Predicted three-dimensional structure of HLA-B*15:03 allele. B) Ramachandran plot of the predicted structure of the HLA-B*15:03 allele.

Figure 6 Schematic presentation of the designed 699 amino acids long multi-epitope vaccine. An adjuvant is added at the amino-terminal with the help of the EAAAK linker (grey). $\mathrm{T}_{\mathrm{h}}$ cell epitopes and B-cell epitopes were joined using GPGPG linkers (red) and CTL epitopes were joined using AAY linkers (Yellow). TH cell, B-cell, and CTL epitopes are depicted with light blue, light green, and sky blue colors respectively.

Figure 7 Predicted secondary structure of the multi-epitope vaccine. 
Figure 8 Predicted solubility of the designed multi-epitope vaccine. QuerySol represents the vaccine and PopAvrSol represents the average solubility (0.45) of E. coli protein.

Figure 9 A) Predicted three-dimensional structure of the multi-epitope vaccine. B) Ramachandran plot of the predicted structure of the multi-epitope vaccine.

572 interfaces of multi-epitope vaccine (magenta) and HLA-B*15:03 (blue) interacting with each

573 other generated using Pymol script. B) Most prominent interacting residues of HLA-B*15:03

574 shown in blue color-forming bonds with the residues of multi-epitope vaccine (magenta).

Figure 11 Distribution of TH cells during vaccination. A) Distribution of memory and 576 non-memory cells. B) Four state distribution of TH cells. C) CD4 T-regulatory lymphocytes

577 count with both total memory and per entity-state counts. D) Different stages of TH cells. E) 578 Percentage of TH cells distribution for three months. TH: T-helper cell, Mem: memory cell, 579 TR: T-helper regulatory cells.

Figure 12 Distribution of CTL during vaccination. A) Distribution of memory and nonmemory cells. B) Four state distribution of TC cells. C) Different states of dendritic cells (DC) 582 including resting, active, and presenting states. The resting state represents the cells not 583 presented to the antigen and anergic state refers to tolerance of T-cells to the antigen due to 584 repeated exposures. TC: T-cytotoxic cell, Mem: Memory cells, and DC: dendritic cells. 
Figure 14 Cytokines, IL-2, and NK cells simulated reaction to vaccination. A) different

589 cells after vaccination showing high fluctuation levels between 310 and 375 cells.

Table 1 Details of selected epitopes based on molecular docking with HLA-B*15:03.

592 after molecular docking.

Table 3 Sequence details of spike glycoprotein sequences of SARS-CoV-2 that were obtained from phylogenetic analysis and were utilized in further analyses.

595

596

Figures

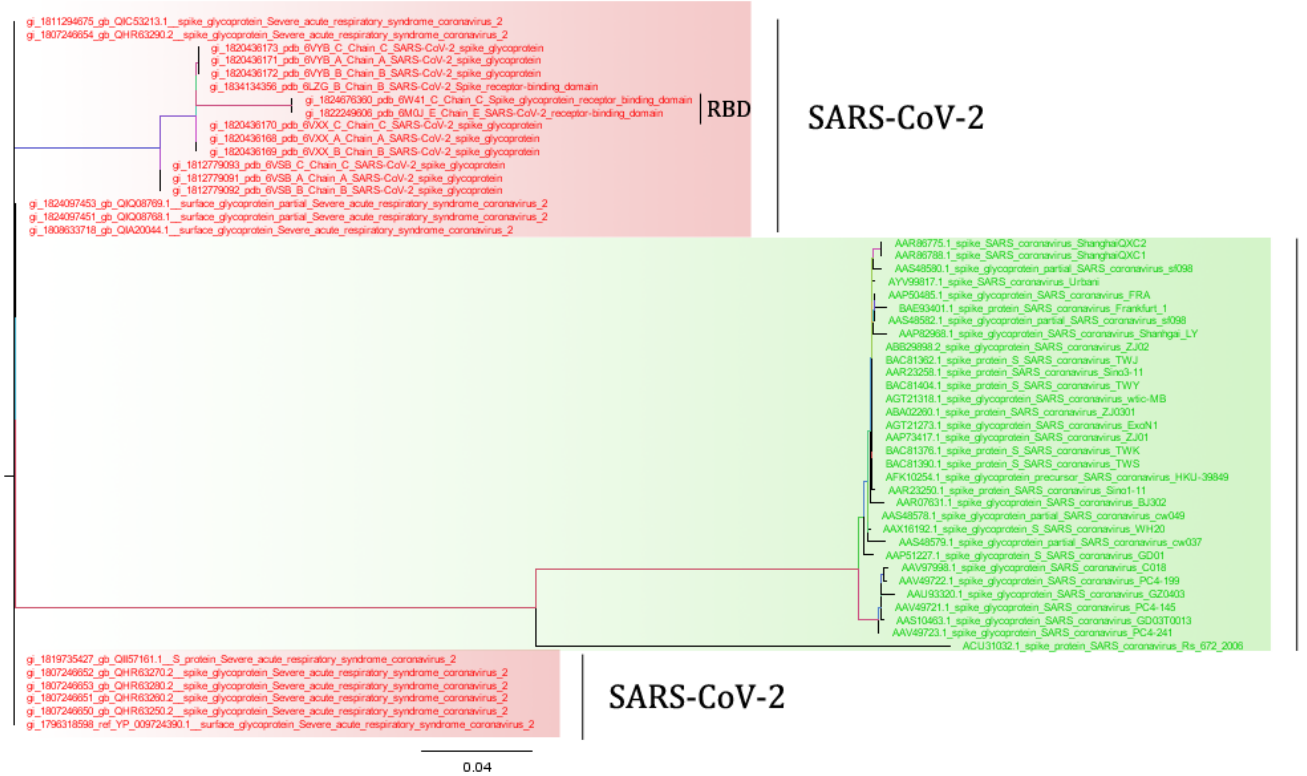

SARS-CoV 
Figure $1 \mathrm{ML}$ tree of spike glycoprotein sequences of SARS-CoV and SARS-CoV-2. Red

601

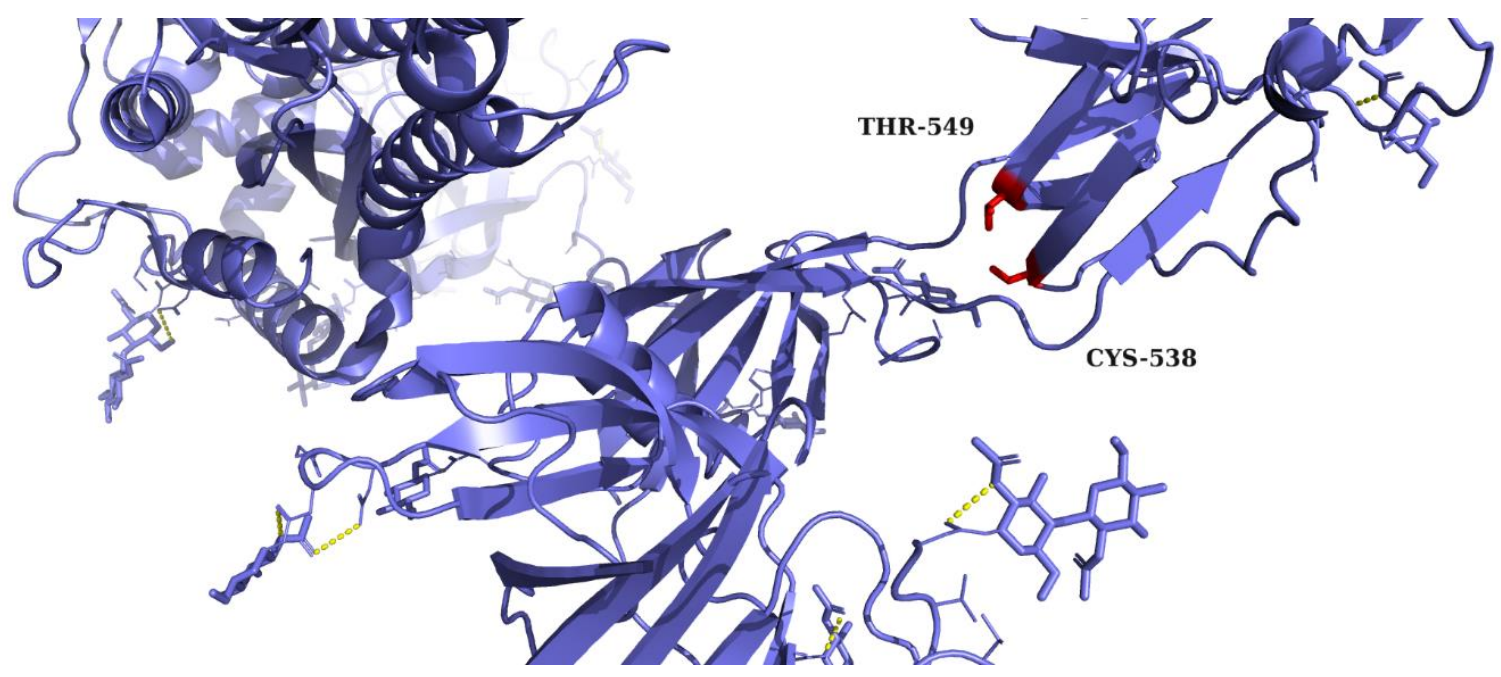

Figure 2 Two sites identified as positively selected mapped on crystal structure SARS- 


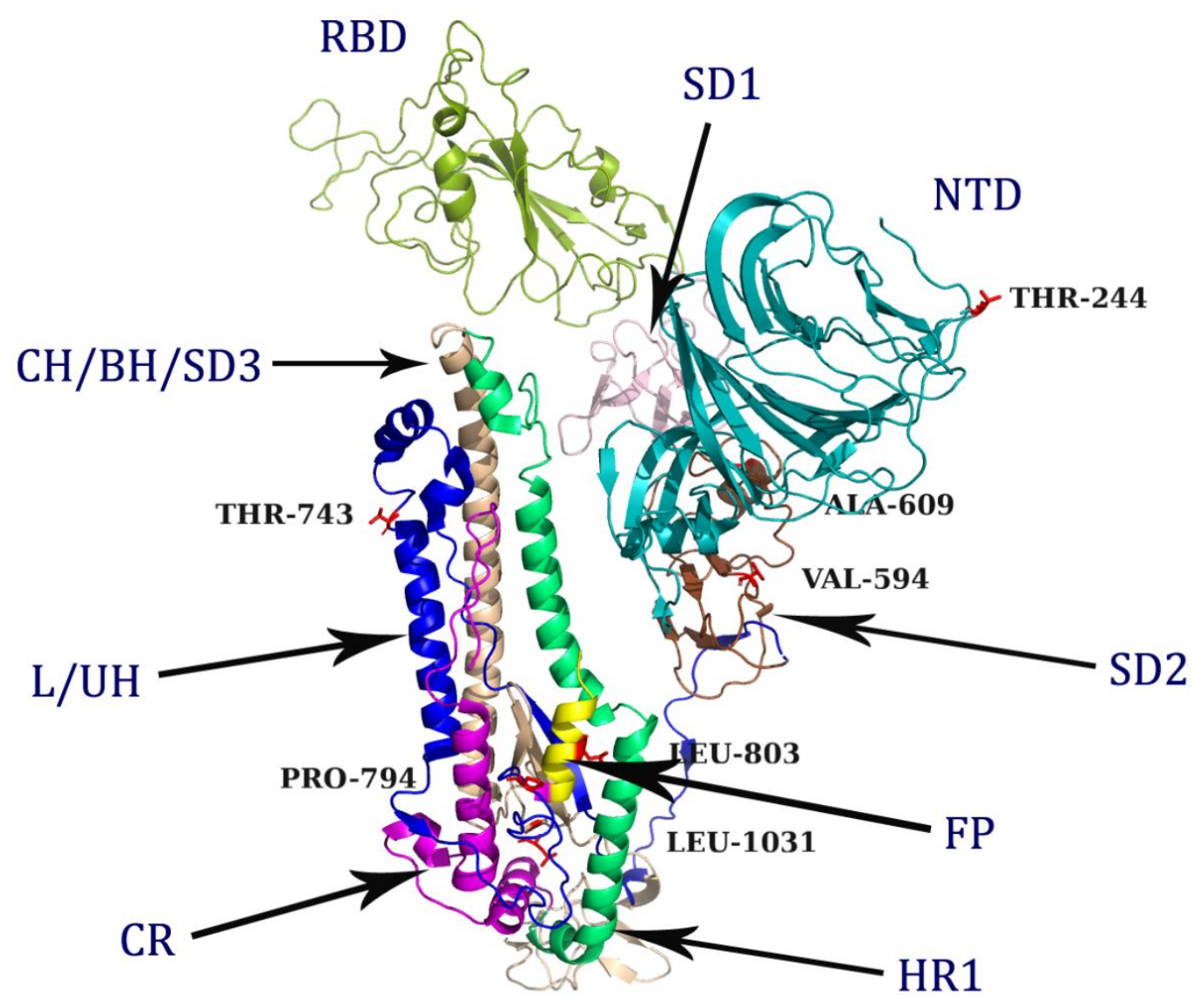

605

Figure 3 Identified positively selected sites (red sticks) mapped on crystal structure

607 SARS-CoV spike glycoprotein (PDB ID: 5X58). Different colors on the structure depict

609 Subdomain1, SD2: Subdomain2, SD3: Subdomain3, CH: Central helix, BH: $\beta$-hairpin, L: 
bioRxiv preprint doi: https://doi.org/10.1101/2020.06.10.143545; this version posted June 14, 2020. The copyright holder for this preprint (which was not certified by peer review) is the author/funder, who has granted bioRxiv a license to display the preprint in perpetuity. It is made available under aCC-BY-NC-ND 4.0 International license.
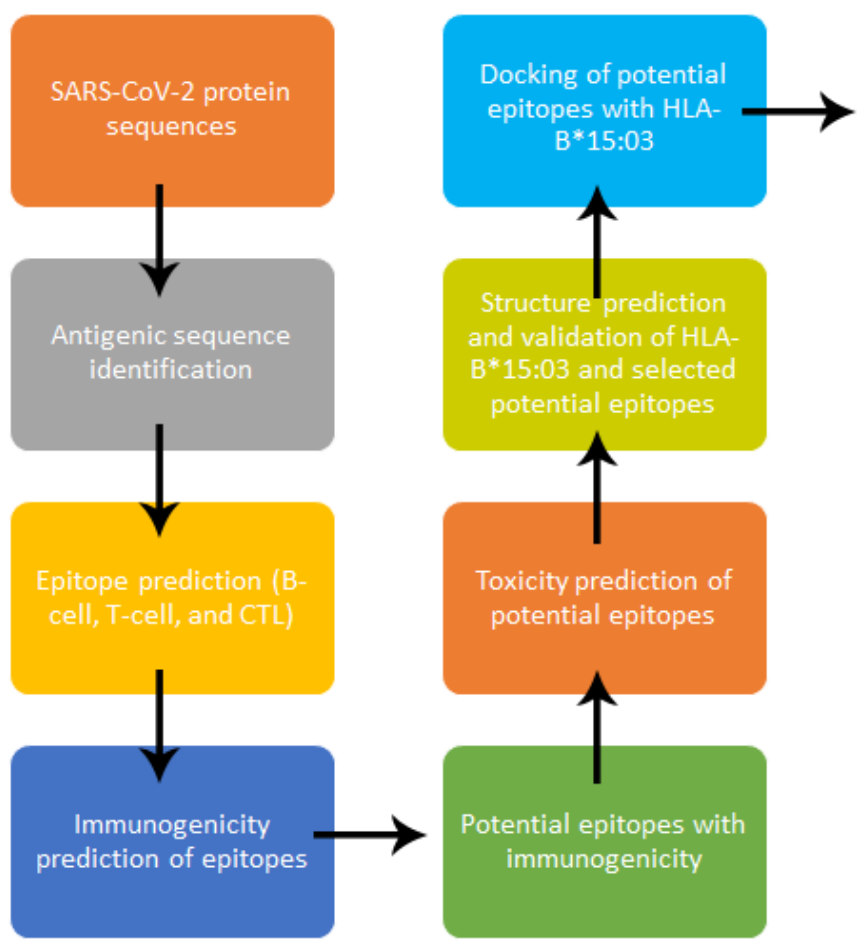

Selection of 5 epitopes

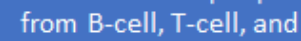

CTL epitopes having

lowest binding affinity

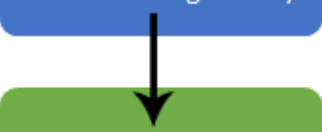

Construction of multi-

epitope vaccine using

linkers and an adjuvant.

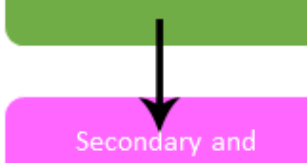

structure prediction of

constructed multi-

epitope vaccine

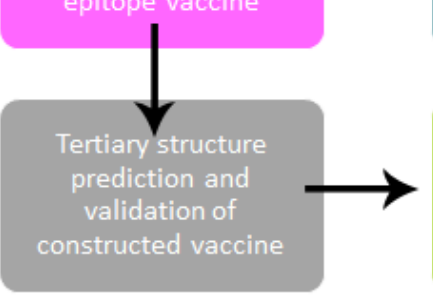

Immune simulation

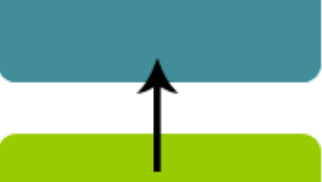

Docking of multi-

epitope vaccine with

HLA-B*15:03

Figure 4 Schema of the methodology followed in the in silico design of a multi-epitope

612 vaccine.

613

614

615

616

617

618 
A

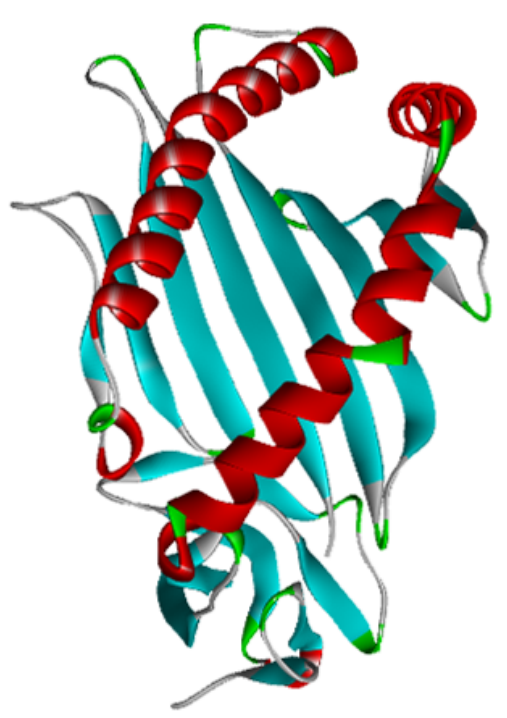

B

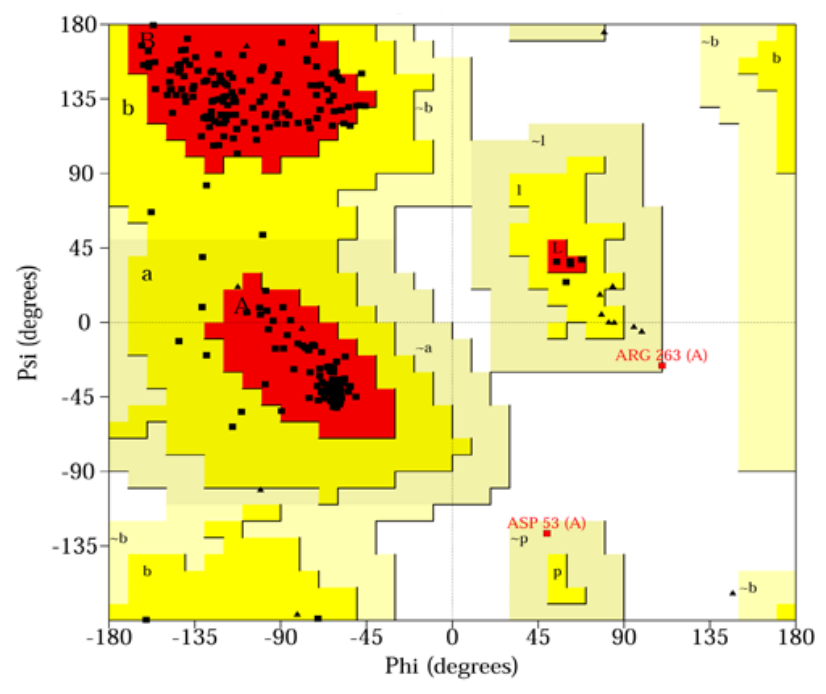

623 Ramachandran plot of the predicted structure of the HLA-B*15:03 allele. 


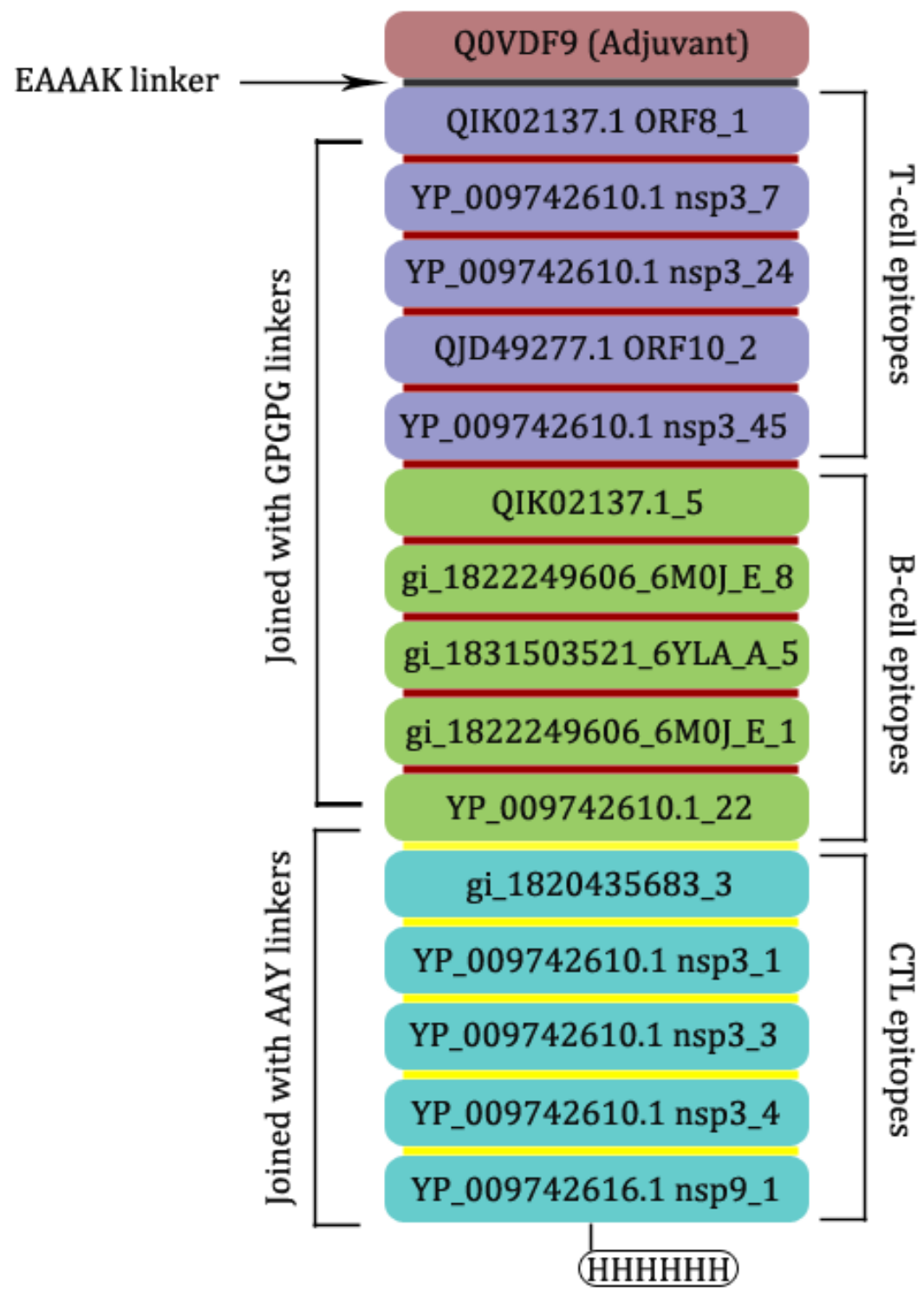

Figure 6 Schematic presentation of the designed 699 amino acids long multi-epitope

626 vaccine. An adjuvant is added at the amino-terminal with the help of the EAAAK linker (grey).

$627 \mathrm{~T}_{\mathrm{h}}$ cell epitopes and B-cell epitopes were joined using GPGPG linkers (red) and CTL epitopes

628 were joined using AAY linkers (Yellow). TH cell, B-cell, and CTL epitopes are depicted with

629 light blue, light green, and sky blue colors respectively. 
bioRxiv preprint doi: https://doi.org/10.1101/2020.06.10.143545; this version posted June 14, 2020. The copyright holder for this preprint (which was not certified by peer review) is the author/funder, who has granted bioRxiv a license to display the preprint in perpetuity. It is made available under aCC-BY-NC-ND 4.0 International license.
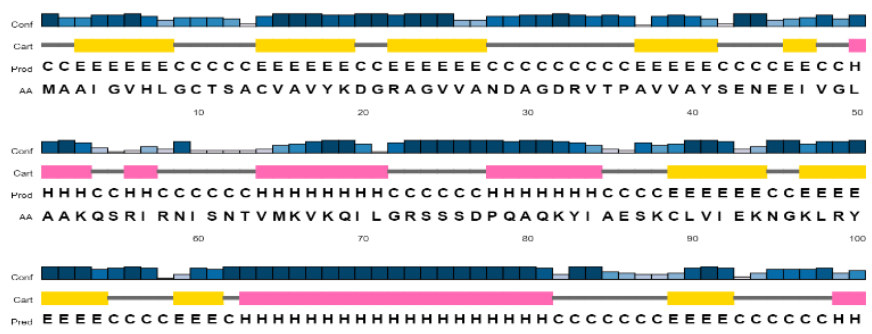

MEI DTGEETKFVNPEDVARLIFSKMKETAHSVLGSDANDVVITVPFDFGEK
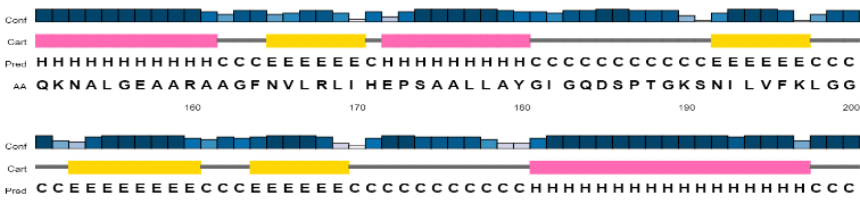

AA TSLSLSVMEVNSGI YRVLSTNTDDNI GGAHFTETLAQYLASEFQRSFKHD
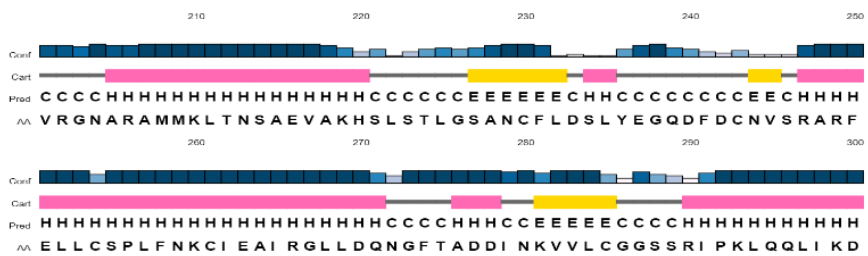

MELLCSPLFNKCIEAI RGLLDQNGFTADDI NKVVLCGGSSRI PKLQQLI KD 310$$
\text { so }
$$

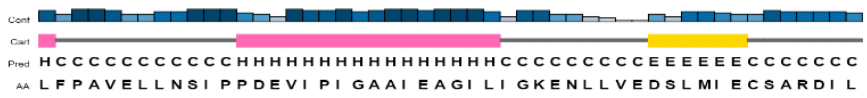

Aa LFPAVELLNI PPDEVI PI GAAIEAGI LI GKENLLVEDSLMI ECSARDIL 300 970

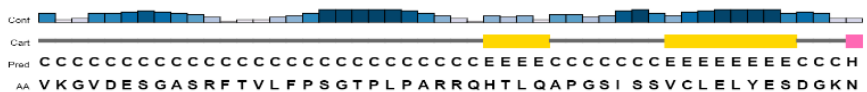
AA VKGVDESGASRFTVLFPSGTPLPARRQHTLQAPGSISSVCLELYESDGKN

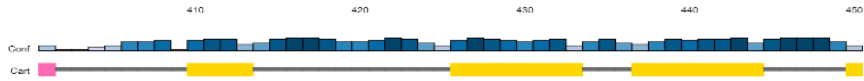

Cot

M SAKEETKFAQVVLQDLDKKENGLRDILAVLTMKRDGSLHVTCTDQETGKC

640

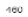

641

642

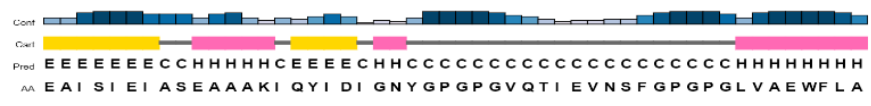

Prod $E E$ EI SI EITASEAAHAKI QYI

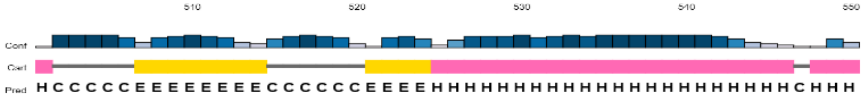

Prad HCCCCCEEEEEEEECCC C C CEEEEHHHHHHHHHHHHHHHHHHHHHCHHH AA YGPGPGYI NVFAFPFGPGPGVVVNAANVYAAYEDFLEYAAYCVNFHHAAY

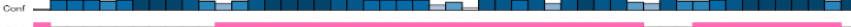

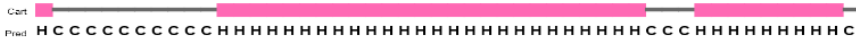
AA SYGFQPTNGVGYQAAYATRFASAAYKTVGELGDAAYNLCPFGEVFAAYFG

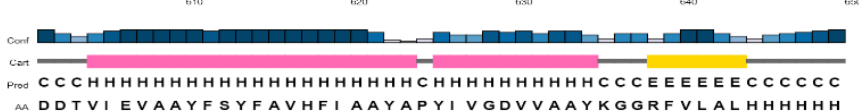

AA DDTVI EVAAYFSYFAVHFI AAYAPYI VGDVVAAYKGGRFVLALHHHHHH

Legend:

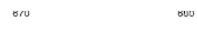

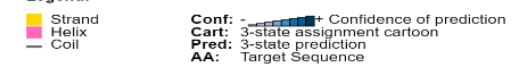




\section{0 \\ Solubility}

650

$\stackrel{\Perp}{\supset} 0.8$

651

652

653

654

0.6

(1)

(0)

(D)

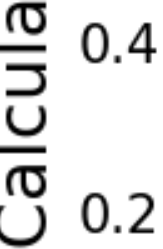

655

656

0.0

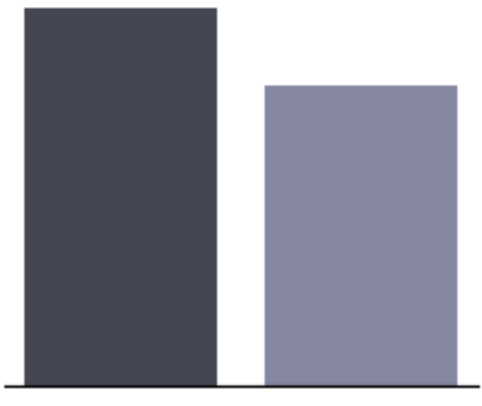

657

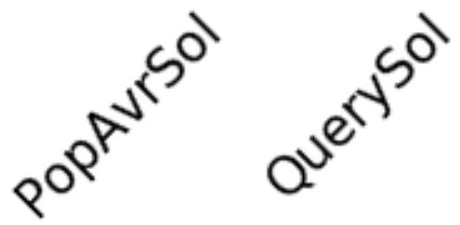

658 


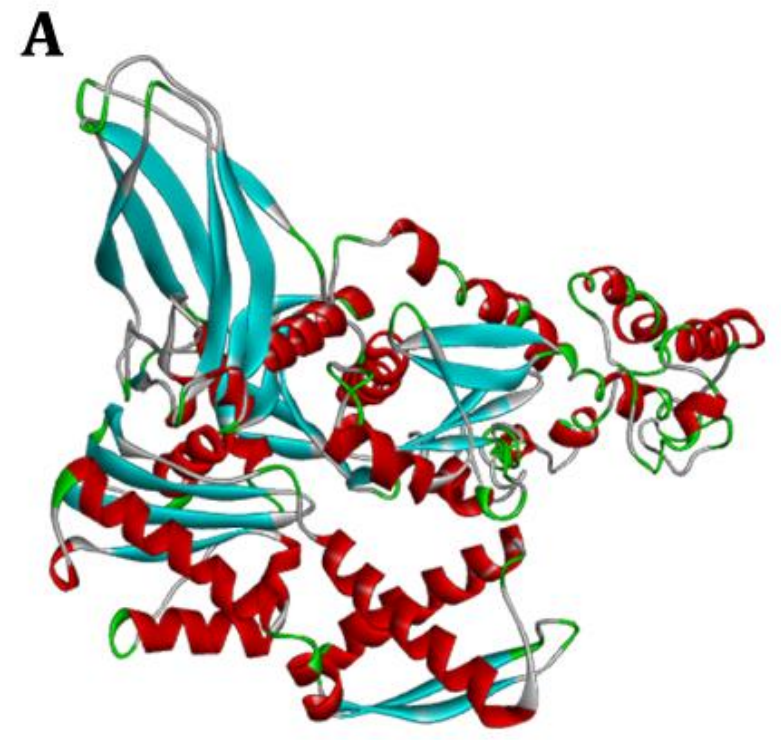

661

Figure 9 A) Predicted three-dimensional structure of the multi-epitope vaccine. B)

Ramachandran plot of the predicted structure of the multi-epitope vaccine.

B

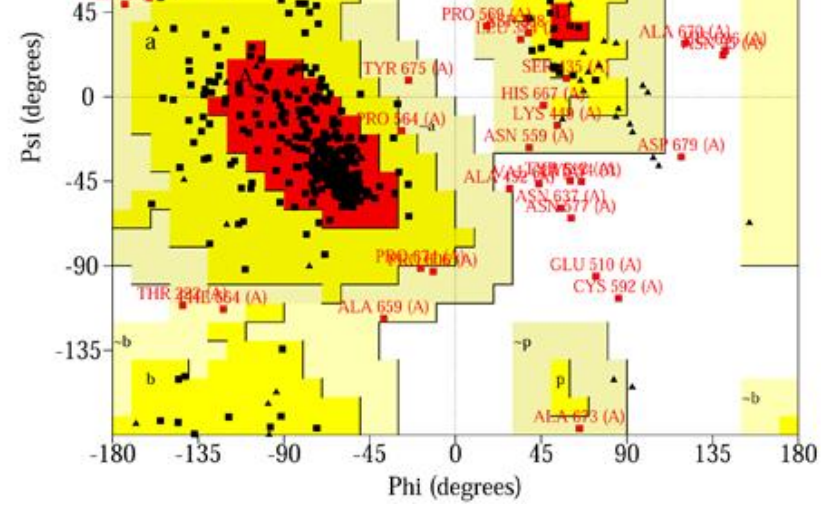

663

662

664

665

666

667

668

669

670

671

672 




B

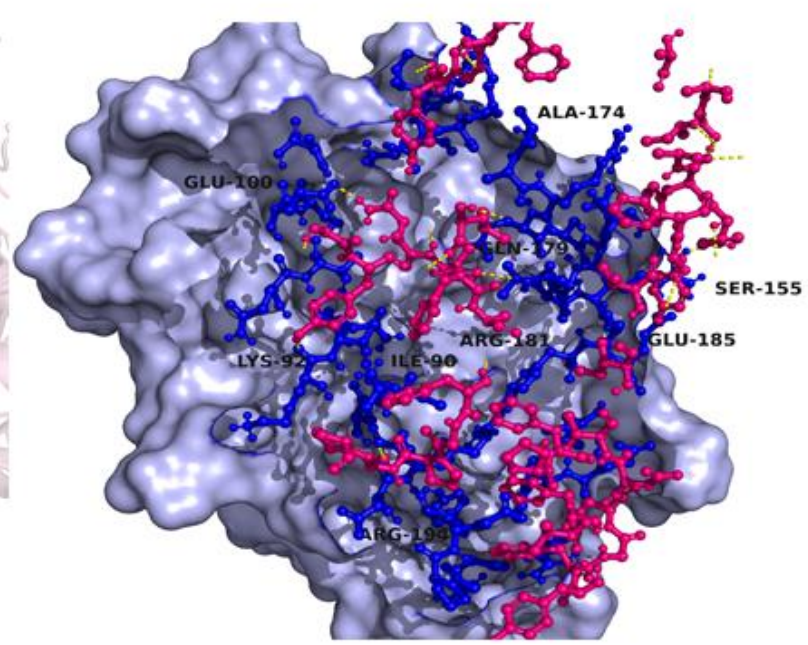

673

Figure 10 Interaction of designed multi-epitope vaccine with HLA-B*15:03 allele. A)

675 interfaces of multi-epitope vaccine (magenta) and HLA-B*15:03 (blue) interacting with each

676 other generated using Pymol script. B) Most prominent interacting residues of HLA-B*15:03

677 shown in blue color-forming bonds with the residues of multi-epitope vaccine (magenta).

678

679

680

681

682

683 
A

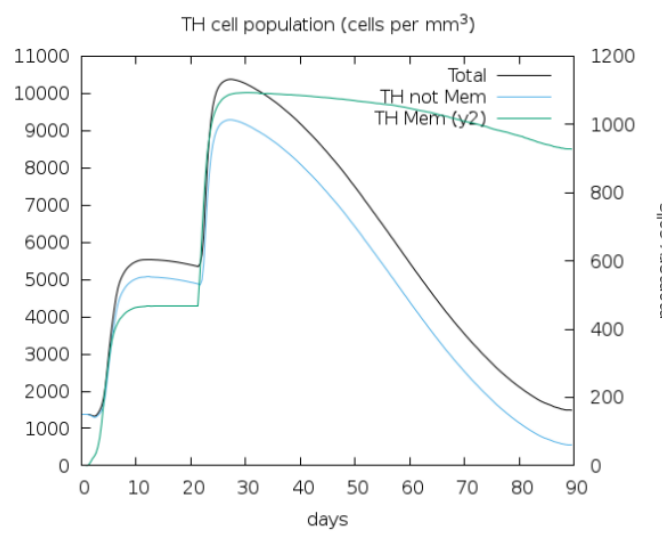

C

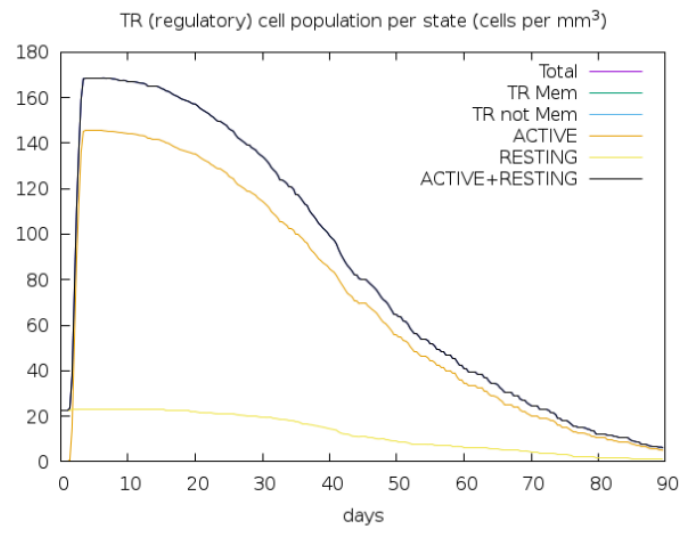

B

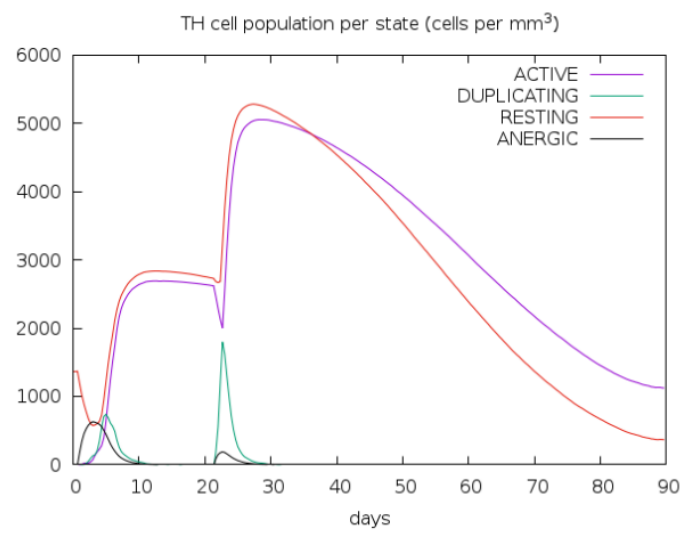

D
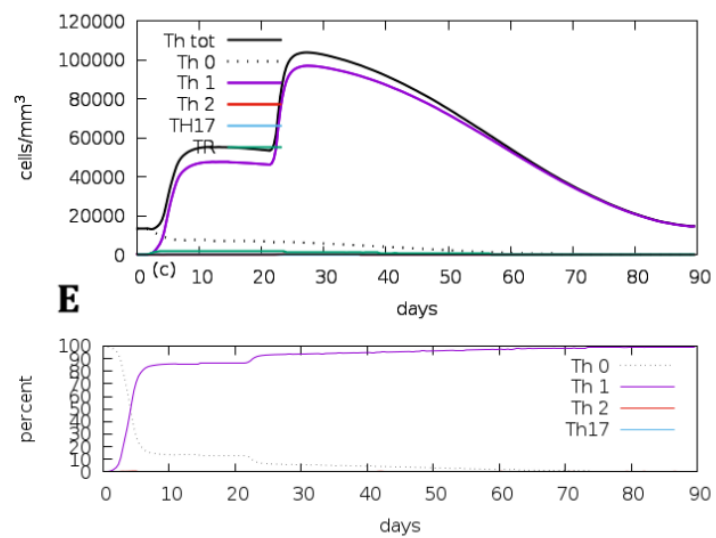

689 Percentage of TH cells distribution for three months. TH: T-helper cell, Mem: memory cell, TR: T-helper regulatory cells. 


\section{A}

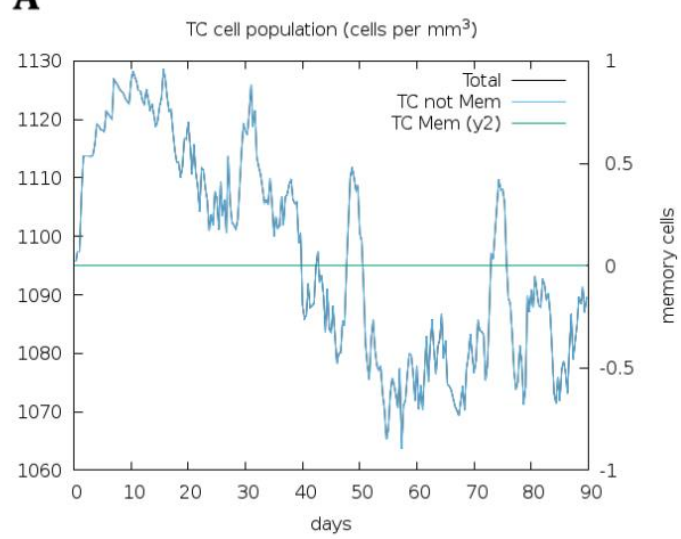

\section{B}

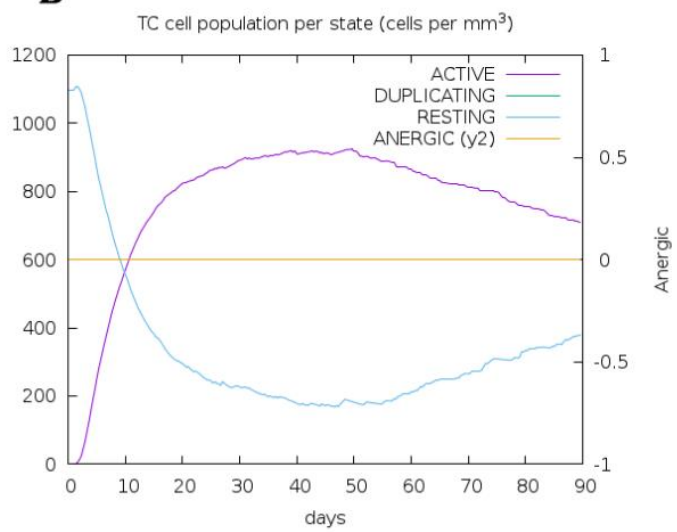

C

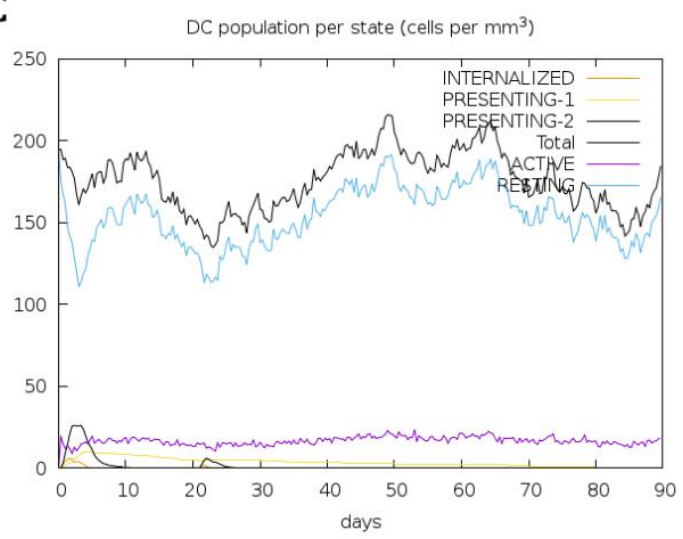

693 
A

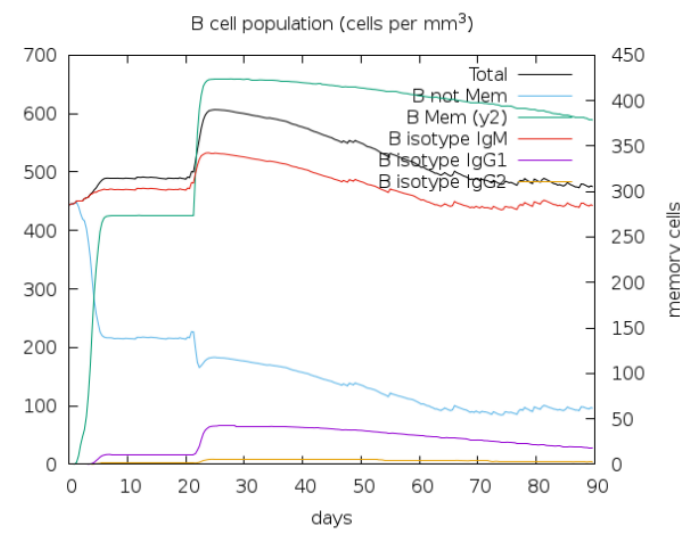

699

700

701

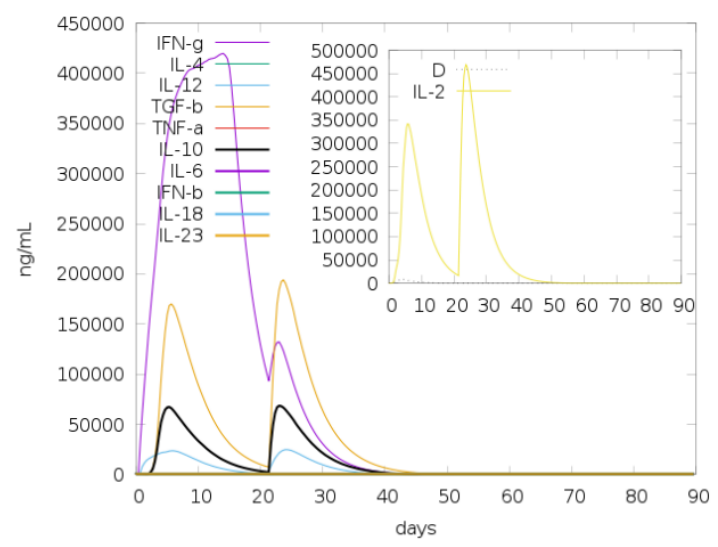

B

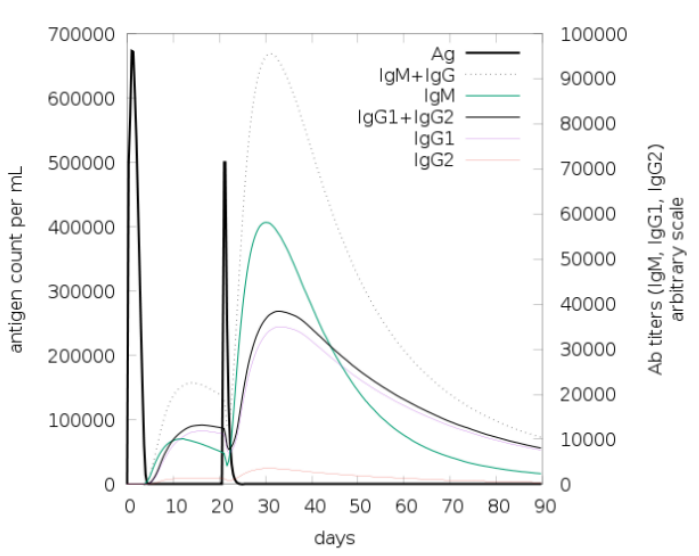

Figure 13 Secondary antibodies response simulation during vaccination. A) formation of different types of B-cells. B) earlier increase in IgM as compared to IgG.

A

703

704

705

706

707
B

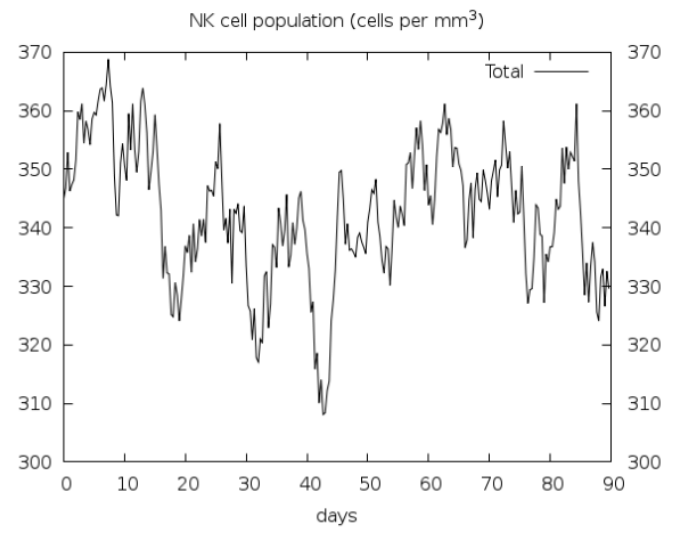

Figure 14 Cytokines, IL-2, and NK cells simulated reaction to vaccination. A) different levels of cytokines through time with a focus on interleukin 2 (IL-2). B) Distributions of NKcells after vaccination showing high fluctuation levels between 310 and 375 cells. 
Table 1 Details of selected epitopes based on molecular docking with HLA-B*15:03.

709

\begin{tabular}{cccccc}
$\begin{array}{c}\text { Type of } \\
\text { epitope }\end{array}$ & Sequence ID & Sequence & Homology & $\begin{array}{c}\text { No of amino acid } \\
\text { residue }\end{array}$ & $\begin{array}{c}\text { Binding } \\
\text { affinity } \\
\text { (kcal/mol) }\end{array}$ \\
& QIK02137.1 ORF8_1 & IQYIDIGNY & 100 & 9 & -6.5 \\
T-Cell & YP_009742610.1 nsp3_7 & VQTIEVNSF & 100 & 9 & -6.5 \\
& YP_009742610.1 nsp3_24 & LVAEWFLAY & 100 & 9 & -6.2 \\
& QJD49277.1 ORF10_2 & YINVFAFPF & 55 & 9 & -6.1 \\
& YP_009742610.1 nsp3_45 & VVVNAANVY & 100 & 9 & -6.1 \\
\multirow{4}{*}{ B-Cell } & QIK02137.1_5 & EDFLEY & 100 & 6 & -8.8 \\
& gi_1822249606_pdb_6M0J_E_8 & CVNFHH & 100 & 6 & -8.8 \\
& gi_1831503521_pdb_6YLA_A_5 & SYGFQPTNGVGYQ & 92 & 13 & -8.1 \\
& gi_1822249606_pdb_6M0J_E_1 & ATRFAS & 66 & 6 & -7.9 \\
& YP_0099742610.1_22 & KTVGELGD & 100 & 8 & -7.8 \\
& gi_1820435683_3 & NLCPFGEVF & 100 & 9 & -7.1 \\
CTL & YP_009742610.1 nsp3_1 & FGDDTVIEV & 100 & 9 & -5.9 \\
& YP_009742610.1 nsp3_3 & FSYFAVHFI & 100 & 9 & -5.1 \\
& YP_009742610.1 nsp3_4 & APYIVGDVV & 100 & 9 & -6.2
\end{tabular}

710

Table 2 All interacting residues of HLA-B*15:03 and multi-epitope vaccine obtained

713 after molecular docking.

\begin{tabular}{cccc}
\multicolumn{2}{c}{ HLA-B*15:03 } & \multicolumn{2}{c}{ Multi-epitope vaccine } \\
$\begin{array}{c}\text { Interacting } \\
\text { residues }\end{array}$ & \#Amino acid & $\begin{array}{c}\text { Interacting } \\
\text { residues }\end{array}$ & \#Amino acid \\
LYS & 92 & TYR & 557 \\
GLN & 89 & PHE & 565 \\
LYS & 170 & TYR & 600 \\
ALA & 174 & TYR & 600 \\
ILE & 90 & PRO & 567 \\
ARG & 175 & ILE & 669 \\
ASP & 85 & PRO & 569 \\
SER & 101 & ASN & 559 \\
GLN & 179 & VAL & 560 \\
GLU & 178 & ALA & 665 \\
THR & 167 & TYR & 602
\end{tabular}




\begin{tabular}{|c|c|c|c|}
\hline SER & 155 & PHE & 664 \\
\hline THR & 93 & PHE & 561 \\
\hline ARG & 86 & PHE & 645 \\
\hline ARG & 175 & PHE & 664 \\
\hline GLU & 190 & ASP & 652 \\
\hline GLU & 82 & PHE & 645 \\
\hline GLN & 96 & TYR & 557 \\
\hline ILE & 166 & TYR & 602 \\
\hline GLN & 179 & ALA & 562 \\
\hline TRP & 191 & THR & 653 \\
\hline TYR & 83 & PHE & 645 \\
\hline ASP & 85 & PRO & 567 \\
\hline TYR & 183 & THR & 653 \\
\hline GLY & 80 & TYR & 648 \\
\hline ARG & 86 & PHE & 641 \\
\hline ASN & 104 & ASN & 559 \\
\hline ARG & 175 & PHE & 661 \\
\hline GLU & 82 & PRO & 640 \\
\hline GLN & 89 & GLY & 568 \\
\hline ALA & 173 & TYR & 602 \\
\hline GLU & 87 & PHE & 645 \\
\hline THR & 93 & ASN & 559 \\
\hline THR & 97 & ASN & 559 \\
\hline THR & 97 & TYR & 557 \\
\hline ALA & 177 & PHE & 664 \\
\hline GLU & 185 & TYR & 660 \\
\hline GLN & 89 & PHE & 561 \\
\hline GLU & 178 & TYR & 660 \\
\hline GLU & 176 & VAL & 560 \\
\hline ARG & 169 & TYR & 602 \\
\hline ASN & 94 & TYR & 557 \\
\hline GLU & 82 & PHE & 641 \\
\hline ALA & 182 & ILE & 655 \\
\hline ALA & 177 & TYR & 660 \\
\hline ALA & 174 & VAL & 560 \\
\hline ARG & 86 & PRO & 567 \\
\hline GLU & 82 & PRO & 567 \\
\hline GLN & 89 & PRO & 567 \\
\hline GLU & 100 & ASN & 559 \\
\hline ARG & 194 & ASP & 651 \\
\hline ARG & 181 & TYR & 663 \\
\hline GLY & 80 & PHE & 649 \\
\hline TYR & 83 & PRO & 567 \\
\hline
\end{tabular}




\begin{tabular}{|c|c|c|c|}
\hline GLU & 178 & VAL & 657 \\
\hline TRP & 191 & ASP & 651 \\
\hline LEU & 187 & ASP & 652 \\
\hline GLN & 179 & PHE & 561 \\
\hline ARG & 181 & PHE & 664 \\
\hline ASP & 85 & GLY & 568 \\
\hline ALA & 173 & PHE & 604 \\
\hline GLU & 178 & PHE & 664 \\
\hline ARG & 175 & ALA & 670 \\
\hline TYR & 183 & TYR & 660 \\
\hline ASN & 94 & ASN & 559 \\
\hline TRP & 191 & PHE & 645 \\
\hline GLU & 190 & ASP & 651 \\
\hline LEU & 180 & VAL & 560 \\
\hline LYS & 170 & TYR & 602 \\
\hline ARG & 194 & PHE & 649 \\
\hline PRO & 81 & TYR & 648 \\
\hline THR & 97 & VAL & 560 \\
\hline ARG & 86 & GLY & 568 \\
\hline GLU & 79 & PHE & 649 \\
\hline GLN & 89 & TYR & 557 \\
\hline GLN & 89 & GLY & 566 \\
\hline THR & 93 & TYR & 557 \\
\hline ARG & 181 & TYR & 660 \\
\hline LEU & 187 & THR & 653 \\
\hline ARG & 175 & ALA & 682 \\
\hline GLN & 179 & VAL & 657 \\
\hline ARG & 194 & GLY & 650 \\
\hline GLU & 82 & VAL & 644 \\
\hline LYS & 170 & ASN & 559 \\
\hline GLN & 89 & PRO & 569 \\
\hline GLU & 100 & GLY & 556 \\
\hline ARG & 175 & ALA & 665 \\
\hline GLU & 178 & ILE & 669 \\
\hline PRO & 81 & PHE & 649 \\
\hline ALA & 182 & TYR & 660 \\
\hline ARG & 86 & THR & 653 \\
\hline LEU & 187 & ILE & 655 \\
\hline TRP & 191 & PHE & 649 \\
\hline GLN & 179 & ILE & 655 \\
\hline TYR & 83 & PHE & 649 \\
\hline TYR & 183 & ILE & 655 \\
\hline GLU & 82 & PHE & \\
\hline
\end{tabular}




\begin{tabular}{|llll}
\hline LEU & 187 & ASP & 651 \\
GLN & 165 & TYR & 602 \\
GLN & 179 & LEU & 549 \\
ALA & 174 & LEU & 549
\end{tabular}

714

Table 3 Sequence details of spike glycoprotein sequences of SARS-CoV-2 that were

716 obtained from phylogenetic analysis and were utilized in further analyses.

\begin{tabular}{|c|c|c|c|c|}
\hline Sr.no & $\begin{array}{c}\text { Sequence ID/ } \\
\text { PDB ID }\end{array}$ & Sequence Name & Chain & Sequence Length \\
\hline 1 & QIC53213.1 & spike_glycoprotein_SARS_corona_virus_2 & - & 1274 \\
\hline 2 & QHR63290.2 & spike_glycoprotein_SARS_corona_virus_2 & - & 1274 \\
\hline 3 & QHR63280.2 & spike_glycoprotein_SARS_corona_virus_2 & - & 1274 \\
\hline 4 & QHR63270.2 & spike_glycoprotein_SARS_corona_virus_2 & - & 1274 \\
\hline 5 & QHR63260.2 & spike_glycoprotein_SARS_corona_virus_2 & - & 1274 \\
\hline 6 & QHR63250.2 & spike_glycoprotein_SARS_corona_virus_2 & - & 1274 \\
\hline 7 & 6VYB & SARS-CoV-2_spike_glycoprotein & C & 1282 \\
\hline 8 & 6VYB & SARS-CoV-2_spike_glycoprotein & B & 1282 \\
\hline 9 & 6VYB & SARS-CoV-2_spike_glycoprotein & A & 1282 \\
\hline 10 & $6 V X X$ & SARS-CoV-2_spike_glycoprotein & $\mathrm{C}$ & 1282 \\
\hline 11 & $6 \mathrm{VXX}$ & SARS-CoV-2_spike_glycoprotein & $\mathrm{B}$ & 1282 \\
\hline 12 & $6 \mathrm{VXX}$ & SARS-CoV-2_spike_glycoprotein & $A$ & 1282 \\
\hline 13 & 6VSB & SARS-CoV-2_spike_glycoprotein & $\mathrm{C}$ & 1289 \\
\hline 14 & 6VSB & SARS-CoV-2_spike_glycoprotein & B & 1289 \\
\hline 15 & 6VSB & SARS-CoV-2_spike_glycoprotein & $A$ & 1289 \\
\hline 16 & 6LZG & SARS-CoV-2_Spike_receptor-binding_domain & B & 210 \\
\hline 17 & $6 W 41$ & Spike_glycoprotein_receptor_binding_domain & C & 232 \\
\hline 18 & QIQ08769.1 & Surface_glycoprotein_partial_SARS_coronavirus & - & 53 \\
\hline 19 & QIQ08768.1 & Surface_glycoprotein_partial_SARS_coronavirus2 & - & 53 \\
\hline 20 & QII57161.1 & S_protein_SARS_coronavirus_2 & - & 1274 \\
\hline 21 & QIA20044.1 & Surface_glycoprotein_SARS_coronavirus2 & - & 1274 \\
\hline 22 & YP_009724390.1 & Surface_glycoprotein_SARS_coronavirus2 & - & 1274 \\
\hline 23 & $6 \mathrm{MOJ}$ & SARS-CoV-2_receptor-binding_domain & $\mathrm{E}$ & 230 \\
\hline
\end{tabular}

